\title{
On Certain 3-Dimensional Limit Boundary Value Problems
}

\author{
V. B. Vasilyev* \\ (Submitted by E. K. Lipachev) \\ Chair of Applied Mathematics and Computer Modeling, Belgorod State National Research University, \\ Belgorod, 308015 Russia
}

Received December 26, 2019; revised January 14, 2020; accepted January 20, 2020

\begin{abstract}
The paper is devoted to studying limit behavior for a solution of model elliptic pseudodifferential equation with some integral boundary condition in 4 -wedge conical canonical 3D singular domain with two parameters. It is shown that the solution of such boundary value problem can have a limit with respect to endpoint values of the parameters in appropriate Sobolev-Slobodetskii space if the boundary function is a solution of a special functional singular integral equation.
\end{abstract}

DOI: $10.1134 / \mathrm{S} 1995080220050133$

Keywords and phrases: elliptic pseudo-differential equation, boundary value problem, singular domain, asymptotic behavior.

\section{INTRODUCTION}

A lot of papers and books are devoted to constructing and developing a theory of elliptic pseudodifferential operators and equations on non-smooth manifolds or on manifolds with non-smooth boundaries [1-5]. The term "a theory" means that these studies describe Fredholm properties and an index theorem.

According to the local principle to obtain Fredholm property for a general pseudo-differential operator we need to study invertibility properties for model operators in so called canonical domains. Distinct model operators and canonical domains generate distinct Fredholm theories.

The author develops its own approach to studying elliptic pseudo-differential equations on manifolds with a non-smooth boundary. Key point of the approach is studying a unique solvability for a model equation in a canonical domains. Such canonical domains can be a whole space $\mathbb{R}^{m}$, a half-space $\mathbb{R}_{+}^{m}=\left\{x \in \mathbb{R}^{m}: x=\left(x^{\prime}, x_{m}\right), x_{m}>0\right\}$ or a certain cone in $\mathbb{R}^{m}$. Some first author's results are included in the book [12], but to now the author have obtained some new results [13, 14, 16, 17] which permit developing the approach more explicitly. Moreover, some results [19, 21] can help to describe more complicated situations than ordinary $m$-dimensional cone in $\mathbb{R}^{m}$, namely we would like to consider here the situation when starting cone degenerates into a cone of a lower dimension.

\section{PRELIMINARIES}

\subsection{Notations}

Here we will introduce some notations and definitions. Let $C_{+}^{a b}$ be a domain inside of a 4 -wedge angle

$$
C_{+}^{a b}=\left\{x \in \mathbb{R}^{3}: x=\left(x_{1}, x_{2}, x_{3}\right), x_{3}>a\left|x_{1}\right|+b\left|x_{2}\right|, a, b>0\right\}
$$

in 3-dimensional space $\mathbb{R}^{3}$. If $A$ is a pseudo-differential operator with a symbol $\tilde{A}(\xi)$ non depending on a spatial variable $x$ then a model pseudo-differential equation under consideration is the following

$$
(A u)(x)=v(x), \quad x \in C_{+}^{a b} .
$$

${ }^{*}$ E-mail: vbv57@inbox.ru 
According to [7] we consider symbols $A(\xi)$ satisfying the condition

$$
c_{1}(1+|\xi|)^{\alpha} \leq|A(\xi)| \leq c_{2}(1+|\xi|)^{\alpha}, \quad \alpha \in \mathbb{R},
$$

with some positive constants $c_{1}, c_{2}$.

The key tool for studying the solvability for the equation (1) is a concept of wave factorization of an elliptic symbol [12]. We need some notations and definitions from [9].

A radial tube domain over the cone $C_{+}^{a b}$ is called a domain of $\mathbb{C}^{3}$ of the following type

$$
T\left(C_{+}^{a b}\right) \equiv\left\{z \in \mathbb{C}^{3}: z=x+i y, x \in \mathbb{R}^{3}, y \in C_{+}^{a b}\right\} .
$$

A conjugate cone $C_{+}^{*}$ is called such a cone for all points of which

$$
x \cdot y>0, \quad \forall y \in C_{+}^{a b},
$$

where $x \cdot y$ means the inner product for $x$ and $y$.

Lemma 1. $C_{+}^{*}=\left\{\xi \in \mathbb{R}^{3}: \xi=\left(\xi_{1}, \xi_{2}, \xi_{3}\right), \xi_{3}>\frac{1}{2 a}\left|\xi_{1}\right|+\frac{1}{2 b}\left|\xi_{2}\right|\right.$.

Proof. This is an easy exercise for undergraduate students.

A cone is called self-conjugate if its conjugate cone coincides with initial one. So, we se that the cone $C_{+}^{a b}$ will be a conjugate if and only if $a=b=\frac{1}{\sqrt{2}}$.

Definition 1. Wave factorization of symbol $\tilde{A}(\xi)$ with respect to the cone $C_{+}^{a b}$ is called its representation in the form

$$
\tilde{A}(\xi)=\tilde{A}_{\neq}(\xi) \tilde{A}_{=}(\xi)
$$

where the factors $\tilde{A}_{\neq}(\xi), \tilde{A}_{=}(\xi)$ satisfy the following conditions:

1) $\tilde{A}_{\neq}(\xi), \tilde{A}_{=}(\xi)$ are defined everywhere without may be the points $\left\{\xi \in \mathbb{R}^{3}: \xi_{3}=\frac{1}{2 a}\left|\xi_{1}\right|+\right.$ $\left.\frac{1}{2 b}\left|\xi_{2}\right|\right\}$;

2) $\tilde{A}_{\neq}(\xi), \tilde{A}_{=}(\xi)$ admit an analytical continuation into radial tube domains $T\left({ }^{*}{ }_{+}^{a b}\right), T\left(-C_{+}^{a b}\right)$ respectively, which satisfy the estimates

$$
\begin{gathered}
\left|A_{\neq}^{ \pm 1}(\xi+i \tau)\right| \leq c_{1}(1+|\xi|+|\tau|)^{ \pm æ}, \\
\left|A_{=}^{ \pm 1}(\xi-i \tau)\right| \leq c_{2}(1+|\xi|+|\tau|)^{ \pm(\alpha-æ)}, \quad \forall \tau \in C_{+}^{*} .
\end{gathered}
$$

The number $æ$ is called index of wave factorization.

Everywhere below we assume that such wave factorization exists.

We study the equation (1) in Sobolev-Slobodetskii space $H^{s}\left(C_{+}^{a b}\right)$. By definition, this space consists of functions $u$ from $H^{s}\left(\mathbb{R}^{m}\right)$ which supports belong to $\overline{C_{+}^{a b}}$. A norm in the space $H^{s}\left(C_{+}^{a b}\right)$ is induced by the $H^{s}$-norm

$$
\|u\|_{s}=\left(\int_{\mathbb{R}^{3}} \tilde{u}(\xi)(1+|\xi|)^{2 s} d \xi\right)^{1 / 2},
$$

where the sign $\sim$ over $u$ denotes its Fourier transform

$$
\tilde{u}(\xi)=\int_{\mathbb{R}^{3}} u\left(x \left(e^{i x \cdot \xi} d x .\right.\right.
$$

The right hand side $v$ in the equation (1) is taken from the space $H_{0}^{s-\alpha}\left(C_{+}^{a b}\right)$ of functions defined in $C_{+}^{a b}$ which admit a continuation $\ell v$ into whole $H^{s-\alpha}\left(\mathbb{R}^{m}\right)$. The norm in such a space is defined as

$$
\|v\|_{s}^{+}=\inf \|\ell v\|_{s}
$$

where inf is taken over all continuations $\ell v$.

After these auxiliaries we can present the construction of a general solution of the equation (1) for certain values $æ$. 


\subsection{A General Solution}

To simplify our constructions we will consider the homogeneous boundary value problem

$$
(A u)(x)=0, \quad x \in C_{+}^{a b} .
$$

We will use our constructions from $[16,17]$ to describe the general solution of the equation (1) for the case $æ-s=1+\delta,|\delta|<1 / 2$.

Let us introduce the following singular integral operators [10, 11]

$$
\begin{aligned}
& \left(S_{1} u\right)\left(\xi_{1}, \xi_{2}, \xi_{3}\right)=v \cdot p \frac{i}{2 \pi} \int_{-\infty}^{+\infty} \frac{u\left(\tau, \xi_{2}, \xi_{3}\right) d \tau}{\xi_{1}-\tau}, \\
& \left(S_{2} u\right)\left(\xi_{1}, \xi_{2}, \xi_{3}\right)=v \cdot p \frac{i}{2 \pi} \int_{-\infty}^{+\infty} \frac{u\left(\xi_{1}, \eta, \xi_{3}\right) d \eta}{\xi_{2}-\eta} .
\end{aligned}
$$

Then

$$
\begin{gathered}
A_{\neq}(\xi) \tilde{u}(\xi)=\tilde{C}_{1}\left(\xi_{1}-a \xi_{3}, \xi_{2}-b \xi_{3}\right)+\tilde{C}_{2}\left(\xi_{1}-a \xi_{3}, \xi_{2}+b \xi_{3}\right) \\
\tilde{+} C_{3}\left(\xi_{1}+a \xi_{3}, \xi_{2}-b \xi_{3}\right)+\tilde{C}_{1}\left(\xi_{1}+a \xi_{3}, \xi_{2}+b \xi_{3}\right),
\end{gathered}
$$

where

$$
\begin{aligned}
\tilde{C}_{1}\left(\xi_{1}-a \xi_{3}, \xi_{2}-b \xi_{3}\right) & =\frac{1}{4} \tilde{c}_{0}\left(\xi_{1}-a \xi_{3}, \xi_{2}-b \xi_{3}\right)-\frac{1}{2}\left(S_{1} \tilde{c}_{0}\right)\left(\xi_{1}-a \xi_{3}, \xi_{2}-b \xi_{3}\right) \\
- & \frac{1}{2}\left(S_{2} \tilde{c}_{0}\right)\left(\xi_{1}-a \xi_{3}, \xi_{2}-b \xi_{3}\right)+\left(S_{1} S_{2} \tilde{c}_{0}\right)\left(\xi_{1}-a \xi_{3}, \xi_{2}-b \xi_{3}\right) \\
\tilde{C}_{2}\left(\xi_{1}-a \xi_{3}, \xi_{2}+b \xi_{3}\right) & =\frac{1}{4} \tilde{c}_{0}\left(\xi_{1}-a \xi_{3}, \xi_{2}+b \xi_{3}\right)-\frac{1}{2}\left(S_{1} \tilde{c}_{0}\right)\left(\xi_{1}-a \xi_{3}, \xi_{2}+b \xi_{3}\right) \\
+ & \frac{1}{2}\left(S_{2} \tilde{c}_{0}\right)\left(\xi_{1}-a \xi_{3}, \xi_{2}+b \xi_{3}\right)-\left(S_{1} S_{2} \tilde{c}_{0}\right)\left(\xi_{1}-a \xi_{3}, \xi_{2}+b \xi_{3}\right) \\
\tilde{C}_{3}\left(\xi_{1}+a \xi_{3}, \xi_{2}-b \xi_{3}\right) & =\frac{1}{4} \tilde{c}_{0}\left(\xi_{1}+a \xi_{3}, \xi_{2}-b \xi_{3}\right)+\frac{1}{2}\left(S_{1} \tilde{c}_{0}\right)\left(\xi_{1}+a \xi_{3}, \xi_{2}-b \xi_{3}\right) \\
- & \frac{1}{2}\left(S_{2} \tilde{c}_{0}\right)\left(\xi_{1}+a \xi_{3}, \xi_{2}-b \xi_{3}\right)-\left(S_{1} S_{2} \tilde{c}_{0}\right)\left(\xi_{1}+a \xi_{3}, \xi_{2}-b \xi_{3}\right) \\
\tilde{C}_{4}\left(\xi_{1}+a \xi_{3}, \xi_{2}+b \xi_{3}\right) & =\frac{1}{4} \tilde{c}_{0}\left(\xi_{1}+a \xi_{3}, \xi_{2}+b \xi_{3}\right)+\frac{1}{2}\left(S_{1} \tilde{c}_{0}\right)\left(\xi_{1}+a \xi_{3}, \xi_{2}+b \xi_{3}\right) \\
+ & \frac{1}{2}\left(S_{2} \tilde{c}_{0}\right)\left(\xi_{1}+a \xi_{3}, \xi_{2}+b \xi_{3}\right)+\left(S_{1} S_{2} \tilde{c}_{0}\right)\left(\xi_{1}+a \xi_{3}, \xi_{2}+b \xi_{3}\right) .
\end{aligned}
$$

Theorem 1. Let $æ-s=1+\delta,|\delta|<1 / 2$. A general solution of the equation (3) is given by the formula (4), where $c_{0}\left(x_{1}, x_{2}\right)$ is an arbitrary function from $H^{s-a e+/ 2}\left(\mathbb{R}^{2}\right)$.

\section{BOUNDARY CONDITIONS}

\subsection{Integral Boundary Condition}

To determine uniquely an arbitrary function $c_{0}\left(\xi_{1}, \xi_{2}\right)$ we need certain additional condition. We suppose that we know the following function $\tilde{u}\left(\xi_{1}, \xi_{2}, 0\right)$. It means that we know the following integral

$$
\int_{-\infty}^{+\infty} u\left(x_{1}, x_{2}, x_{3}\right) d x_{3} \equiv g\left(x_{1}, x_{2}\right),
$$

the condition (5) has the following form in the Fourier image

$$
\tilde{u}\left(\xi_{1}, \xi_{2}, 0\right)=\tilde{g}\left(\xi_{1}, \xi_{2}\right) .
$$




\subsection{Solution of a Boundary Value Problem}

Substituting (6) into (4) we can collect similar summands. Indeed, we have

$$
\begin{aligned}
\tilde{A}_{\neq}(\xi) \tilde{u}(\xi)= & \sum_{k=1}^{4} \tilde{C}_{k}\left(\xi_{1}, \xi_{2}\right)=\frac{1}{4} \tilde{c}_{0}\left(\xi_{1}, \xi_{2}\right)-\frac{1}{2}\left(S_{1} \tilde{c}_{0}\right)\left(\xi_{1}, \xi_{2}\right)-\frac{1}{2}\left(S_{2} \tilde{c}_{0}\right)\left(\xi_{1}, \xi_{2}\right)+\left(S_{1} S_{2} \tilde{c}_{0}\right)\left(\xi_{1}, \xi_{2}\right) \\
& +\frac{1}{4} \tilde{c}_{0}\left(\xi_{1}, \xi_{2}\right)-\frac{1}{2}\left(S_{1} \tilde{c}_{0}\right)\left(\xi_{1}, \xi_{2}\right)+\frac{1}{2}\left(S_{2} \tilde{c}_{0}\right)\left(\xi_{1}, \xi_{2}\right)-\left(S_{1} S_{2} \tilde{c}_{0}\right)\left(\xi_{1}, \xi_{2}\right) \\
& +\frac{1}{4} \tilde{c}_{0}\left(\xi_{1}, \xi_{2}\right)+\frac{1}{2}\left(S_{1} \tilde{c}_{0}\right)\left(\xi_{1}, \xi_{2}\right)-\frac{1}{2}\left(S_{2} \tilde{c}_{0}\right)\left(\xi_{1}, \xi_{2}\right)-\left(S_{1} S_{2} \tilde{c}_{0}\right)\left(\xi_{1}, \xi_{2}\right) \\
+ & \frac{1}{4} \tilde{c}_{0}\left(\xi_{1}, \xi_{2}\right)+\frac{1}{2}\left(S_{1} \tilde{c}_{0}\right)\left(\xi_{1}, \xi_{2}\right)+\frac{1}{2}\left(S_{2} \tilde{c}_{0}\right)\left(\xi_{1}, \xi_{2}\right)+\left(S_{1} S_{2} \tilde{c}_{0}\right)\left(\xi_{1}, \xi_{2}\right)=\tilde{c}_{0}\left(\xi_{1}, \xi_{2}\right) .
\end{aligned}
$$

Substituting the condition (6) into latter result we have the following

$$
\tilde{A}_{\neq}\left(\xi^{\prime}, 0\right) \tilde{u}\left(\xi^{\prime}, 0\right)=\tilde{c}_{0}\left(\xi^{\prime}\right), \quad \xi^{\prime}-\left(\xi_{1}, \xi_{2}\right) .
$$

Therefore

$$
\tilde{c}_{0}\left(\xi^{\prime}\right)=\tilde{A}_{\neq}\left(\xi^{\prime}, 0\right) \tilde{g}\left(\xi^{\prime}\right) .
$$

Theorem 2. Let $æ-s=1+\delta,|\delta|<1 / 2, g \in H^{s+1 / 2}\left(\mathbb{R}^{2}\right)$. The unique solution of the boundary value problem (3), (5) is given by the formula (4), where $c_{0}\left(x_{1}, x_{2}\right)$ is defined by the formula (7).

\section{LIMIT BEHAVIOR}

\subsection{The Case $a \rightarrow 0, b \rightarrow 0$}

This case corresponds to the half-space $\xi_{3}>0$. Thus we have according to the formula (4)

$$
\tilde{A}_{\neq}(\xi) \tilde{u}(\xi)=\sum_{k=1}^{4} \tilde{C}_{k}\left(\xi_{1}, \xi_{2}\right)=\tilde{c}_{0}\left(\xi_{1}, \xi_{2}\right) .
$$

Thus, we have the same formula for a general solution as in [7]. Let us note that for this case the wave factorization transform into ordinary factorization with a parameter with respect to the one-dimensional cone $(0,+\infty)$.

$$
\text { 4.2. The Case } a \rightarrow 0, b=\text { const }
$$

$$
\begin{gathered}
\tilde{C}_{1}\left(\xi_{1}, \xi_{2}-b \xi_{3}\right)=\frac{1}{4} \tilde{c}_{0}\left(\xi_{1}, \xi_{2}-b \xi_{3}\right)-\frac{1}{2}\left(S_{1} \tilde{c}_{0}\right)\left(\xi_{1}, \xi_{2}-b \xi_{3}\right) \\
-\frac{1}{2}\left(S_{2} \tilde{c}_{0}\right)\left(\xi_{1}, \xi_{2}-b \xi_{3}\right)+\left(S_{1} S_{2} \tilde{c}_{0}\right)\left(\xi_{1}, \xi_{2}-b \xi_{3}\right) ; \\
\tilde{C}_{2}\left(\xi_{1}, \xi_{2}+b \xi_{3}\right)=\frac{1}{4} \tilde{c}_{0}\left(\xi_{1}, \xi_{2}+b \xi_{3}\right)-\frac{1}{2}\left(S_{1} \tilde{c}_{0}\right)\left(\xi_{1}, \xi_{2}+b \xi_{3}\right) \\
+\frac{1}{2}\left(S_{2} \tilde{c}_{0}\right)\left(\xi_{1}, \xi_{2}+b \xi_{3}\right)-\left(S_{1} S_{2} \tilde{c}_{0}\right)\left(\xi_{1}, \xi_{2}+b \xi_{3}\right) ; \\
\tilde{C}_{3}\left(\xi_{1}, \xi_{2}-b \xi_{3}\right)=\frac{1}{4} \tilde{c}_{0}\left(\xi_{1}, \xi_{2}-b \xi_{3}\right)+\frac{1}{2}\left(S_{1} \tilde{c}_{0}\right)\left(\xi_{1}, \xi_{2}-b \xi_{3}\right) \\
-\frac{1}{2}\left(S_{2} \tilde{c}_{0}\right)\left(\xi_{1}, \xi_{2}-b \xi_{3}\right)-\left(S_{1} S_{2} \tilde{c}_{0}\right)\left(\xi_{1}, \xi_{2}-b \xi_{3}\right) ; \\
\tilde{C}_{4}\left(\xi_{1}, \xi_{2}+b \xi_{3}\right)=\frac{1}{4} \tilde{c}_{0}\left(\xi_{1}, \xi_{2}+b \xi_{3}\right)+\frac{1}{2}\left(S_{1} \tilde{c}_{0}\right)\left(\xi_{1}, \xi_{2}+b \xi_{3}\right)
\end{gathered}
$$




$$
+\frac{1}{2}\left(S_{2} \tilde{c}_{0}\right)\left(\xi_{1}, \xi_{2}+b \xi_{3}\right)+\left(S_{1} S_{2} \tilde{c}_{0}\right)\left(\xi_{1}, \xi_{2}+b \xi_{3}\right) .
$$

For this case the formula (4) becomes the following

$$
\tilde{A}_{\neq}(\xi) \tilde{u}(\xi)=\frac{\tilde{c}_{0}\left(\xi_{1}, \xi_{2}-b \xi_{3}\right)+\tilde{c}_{0}\left(\xi_{1}, \xi_{2}+b \xi_{3}\right)}{2}+\left(S_{2} \tilde{c}_{0}\right)\left(\xi_{1}, \xi_{2}+b \xi_{3}\right)-\left(S_{2} \tilde{c}_{0}\right)\left(\xi_{1}, \xi_{2}-b \xi_{3}\right) .
$$

Taking into account the formula (7) we can write

$$
\begin{array}{r}
\tilde{A}_{\neq}(\xi) \tilde{u}(\xi)=\frac{\tilde{A}_{\neq}\left(\xi_{1}, \xi_{2}-b \xi_{3}, 0\right) \tilde{g}\left(\xi_{1}, \xi_{2}-b \xi_{3}\right)+\tilde{A}_{\neq}\left(\xi_{1}, \xi_{2}+b \xi_{3}, 0\right) \tilde{g}\left(\xi_{1}, \xi_{2}+b \xi_{3}\right)}{2} \\
+\frac{i}{2 \pi}\left(v \cdot p \cdot \int_{-\infty}^{+\infty} \frac{\tilde{A}_{\neq}\left(\xi_{1}, \eta, 0\right) \tilde{g}\left(\xi_{1}, \eta\right) d \eta}{\xi_{2}+b \xi_{3}-\eta}-v \cdot p \cdot \int_{-\infty}^{+\infty} \frac{\tilde{A}_{\neq}\left(\xi_{1}, \eta, 0\right) \tilde{g}\left(\xi_{1}, \eta\right) d \eta}{\xi_{2}-b \xi_{3}-\eta}\right) .
\end{array}
$$

\subsection{The Case $a=$ const, $b \rightarrow 0$}

This case is analogical to the previous one, and we have the following representation

$$
\begin{gathered}
\tilde{C}_{1}\left(\xi_{1}-a \xi_{3}, \xi_{2}\right)=\frac{1}{4} \tilde{c}_{0}\left(\xi_{1}-a \xi_{3}, \xi_{2}\right)-\frac{1}{2}\left(S_{1} \tilde{c}_{0}\right)\left(\xi_{1}-a \xi_{3}, \xi_{2}\right) \\
-\frac{1}{2}\left(S_{2} \tilde{c}_{0}\right)\left(\xi_{1}-a \xi_{3}, \xi_{2}\right)+\left(S_{1} S_{2} \tilde{c}_{0}\right)\left(\xi_{1}-a \xi_{3}, \xi_{2}\right) ; \\
\tilde{C}_{2}\left(\xi_{1}-a \xi_{3}, \xi_{2}\right)=\frac{1}{4} \tilde{c}_{0}\left(\xi_{1}-a \xi_{3}, \xi_{2}\right)-\frac{1}{2}\left(S_{1} \tilde{c}_{0}\right)\left(\xi_{1}-a \xi_{3}, \xi_{2}\right) \\
+\frac{1}{2}\left(S_{2} \tilde{c}_{0}\right)\left(\xi_{1}-a \xi_{3}, \xi_{2}\right)-\left(S_{1} S_{2} \tilde{c}_{0}\right)\left(\xi_{1}-a \xi_{3}, \xi_{2}\right) ; \\
\tilde{C}_{3}\left(\xi_{1}+a \xi_{3}, \xi_{2}\right)=\frac{1}{4} \tilde{c}_{0}\left(\xi_{1}+a \xi_{3}, \xi_{2}\right)+\frac{1}{2}\left(S_{1} \tilde{c}_{0}\right)\left(\xi_{1}+a \xi_{3}, \xi_{2}\right) \\
-\frac{1}{2}\left(S_{2} \tilde{c}_{0}\right)\left(\xi_{1}+a \xi_{3}, \xi_{2}\right)-\left(S_{1} S_{2} \tilde{c}_{0}\right)\left(\xi_{1}+a \xi_{3}, \xi_{2}\right) ; \\
\tilde{C}_{4}\left(\xi_{1}+a \xi_{3}, \xi_{2}\right)=\frac{1}{4} \tilde{c}_{0}\left(\xi_{1}+a \xi_{3}, \xi_{2}\right)+\frac{1}{2}\left(S_{1} \tilde{c}_{0}\right)\left(\xi_{1}+a \xi_{3}, \xi_{2}\right) \\
+\frac{1}{2}\left(S_{2} \tilde{c}_{0}\right)\left(\xi_{1}+a \xi_{3}, \xi_{2}\right)+\left(S_{1} S_{2} \tilde{c}_{0}\right)\left(\xi_{1}+a \xi_{3}, \xi_{2}\right) .
\end{gathered}
$$

For this case the formula (4) becomes the following

$$
\tilde{A}_{\neq}(\xi) \tilde{u}(\xi)=\frac{\tilde{c}_{0}\left(\xi_{1}-a \xi_{3}, \xi_{2}\right)+\tilde{c}_{0}\left(\xi_{1}+a \xi_{3}, \xi_{2}\right)}{2}+\left(S_{1} \tilde{c}_{0}\right)\left(\xi_{1}+a \xi_{3}, \xi_{2}\right)-\left(S_{1} \tilde{c}_{0}\right)\left(\xi_{1}-a \xi_{3}, \xi_{2}\right) .
$$

Thus, according to (7) we obtain

$$
\begin{aligned}
& \tilde{A}_{\neq}(\xi) \tilde{u}(\xi)=\frac{\tilde{A}_{\neq}\left(\xi_{1}-a \xi_{3}, \xi_{2}, 0\right) \tilde{g}\left(\xi_{1}-a \xi_{3}, \xi_{2}\right)+\tilde{A}_{\neq}\left(\xi_{1}+a \xi_{3}, \xi_{2}, 0\right) \tilde{g}\left(\xi_{1}+a \xi_{3}, \xi_{2}\right)}{2} \\
&+\frac{i}{2 \pi}\left(v \cdot p \cdot \int_{-\infty}^{+\infty} \frac{\tilde{A}_{\neq}\left(\eta, \xi_{2}, 0\right) \tilde{g}\left(\eta, \xi_{2}\right) d \eta}{\xi_{1}+a \xi_{3}-\eta}-v \cdot p \cdot \int_{-\infty}^{+\infty} \frac{\tilde{A}_{\neq}\left(\eta, \xi_{2}, 0\right) \tilde{g}\left(\eta, \xi_{2}\right) d \eta}{\xi_{1}-a \xi_{3}-\eta}\right) .
\end{aligned}
$$

Remark 1. It seems very interesting that the formulas (8), (9) are very close to formulas given in [6]. 


\subsection{The Case $a \rightarrow \infty, b=$ const}

This is more complicated case in comparison with the previous ones. Really, let us introduce the change of variables $\xi_{1}-a \xi_{3}=t_{1}, \xi_{1}+a \xi_{3}=t_{3}$. Then we obtain

$$
\xi_{1}=\frac{t_{3}+t_{1}}{2}, \quad \xi_{3}=\frac{t_{3}-t_{1}}{2 a} .
$$

So we see that $\xi_{3} \rightarrow 0$ as $a \rightarrow \infty$. According to this fact we calculate $\tilde{C}_{k}, k=1,2,3,4$ under $a \rightarrow \infty$

$$
\begin{aligned}
& \tilde{C}_{1}\left(t_{1}, \xi_{2}\right)=\frac{1}{4} \tilde{c}_{0}\left(t_{1}, \xi_{2}\right)-\frac{1}{2}\left(S_{1} \tilde{c}_{0}\right)\left(t_{1}, \xi_{2}\right)-\frac{1}{2}\left(S_{2} \tilde{c}_{0}\right)\left(t_{1}, \xi_{2}\right)+\left(S_{1} S_{2} \tilde{c}_{0}\right)\left(t_{1}, \xi_{2}\right) ; \\
& \tilde{C}_{2}\left(t_{1}, \xi_{2}\right)=\frac{1}{4} \tilde{c}_{0}\left(t_{1}, \xi_{2}\right)-\frac{1}{2}\left(S_{1} \tilde{c}_{0}\right)\left(t_{1}, \xi_{2}\right)+\frac{1}{2}\left(S_{2} \tilde{c}_{0}\right)\left(t_{1}, \xi_{2}\right)-\left(S_{1} S_{2} \tilde{c}_{0}\right)\left(t_{1}, \xi_{2}\right) ; \\
& \tilde{C}_{3}\left(t_{3}, \xi_{2}\right)=\frac{1}{4} \tilde{c}_{0}\left(t_{3}, \xi_{2}\right)+\frac{1}{2}\left(S_{1} \tilde{c}_{0}\right)\left(t_{3}, \xi_{2}\right)-\frac{1}{2}\left(S_{2} \tilde{c}_{0}\right)\left(t_{3}, \xi_{2}\right)-\left(S_{1} S_{2} \tilde{c}_{0}\right)\left(t_{3}, \xi_{2}\right) ; \\
& \tilde{C}_{4}\left(t_{3}, \xi_{2}\right)=\frac{1}{4} \tilde{c}_{0}\left(t_{3}, \xi_{2}\right)+\frac{1}{2}\left(S_{1} \tilde{c}_{0}\right)\left(t_{3}, \xi_{2}\right)+\frac{1}{2}\left(S_{2} \tilde{c}_{0}\right)\left(t_{3}, \xi_{2}\right)+\left(S_{1} S_{2} \tilde{c}_{0}\right)\left(t_{3}, \xi_{2}\right) .
\end{aligned}
$$

Taking into account the formula (4) we have for new variables $t_{1}, \xi_{2}, t_{3}, a \rightarrow \infty$ the following equality

$$
\left.\tilde{A}_{\neq}\left(\frac{t_{3}+t_{1}}{2}, \xi_{2}, 0\right) \tilde{u}\left(\frac{t_{3}+t_{1}}{2}, \xi_{2}, 0\right)=\frac{\tilde{c}_{0}\left(t_{3}, \xi_{2}\right)+\tilde{c}_{0}\left(t_{1}, \xi_{2}\right)}{2}+\left(\left(S_{1} \tilde{c}_{0}\right)\left(t_{3}, \xi_{2}\right)-S_{1} \tilde{c}_{0}\right)\left(t_{1}, \xi_{2}\right)\right)
$$

and using the condition (6) we obtain

$$
\left.\tilde{A}_{\neq}\left(\frac{t_{3}+t_{1}}{2}, \xi_{2}, 0\right) \tilde{g}\left(\frac{t_{3}+t_{1}}{2}, \xi_{2}\right)=\frac{\tilde{c}_{0}\left(t_{3}, \xi_{2}\right)+\tilde{c}_{0}\left(t_{1}, \xi_{2}\right)}{2}+\left(\left(S_{1} \tilde{c}_{0}\right)\left(t_{3}, \xi_{2}\right)-S_{1} \tilde{c}_{0}\right)\left(t_{1}, \xi_{2}\right)\right) .
$$

Substituting the formula (7) into the latter equation we finally obtain

$$
\begin{aligned}
\tilde{A}_{\neq} & \left(\frac{t_{3}+t_{1}}{2}, \xi_{2}, 0\right) \tilde{g}\left(\frac{t_{3}+t_{1}}{2}, \xi_{2}\right)=\frac{\tilde{A}_{\neq}\left(t_{3}, \xi_{2}, 0\right) \tilde{g}\left(t_{3}, \xi_{2}\right)+\tilde{A}_{\neq}\left(t_{1}, \xi_{2}, 0\right) \tilde{g}\left(t_{1}, \xi_{2}\right)}{2} \\
& +\frac{i}{2 \pi}\left(v \cdot p \cdot \int_{-\infty}^{+\infty} \frac{\tilde{A}_{\neq}\left(\eta, \xi_{2}, 0\right) \tilde{g}\left(\eta, \xi_{2}\right) d \eta}{t_{3}-\eta}-v \cdot p \cdot \int_{-\infty}^{+\infty} \frac{\tilde{A}_{\neq}\left(\eta, \xi_{2}, 0\right) \tilde{g}\left(\eta, \xi_{2}\right) d \eta}{t_{1}-\eta}\right) .
\end{aligned}
$$

This equality ( 8 ) is one-dimensional functional singular integral equation with a parameter with respect to the function $\tilde{g}\left(\xi_{1}, \xi_{2}\right)$.

\subsection{The Case $a=$ const, $b \rightarrow \infty$}

This case is very similar to the previous one but nevertheless we will describe the corresponding formulas and equations. A structure of the solution is defined by the following functions

$$
\begin{aligned}
& \tilde{C}_{1}\left(\xi_{1}, t_{2}\right)=\frac{1}{4} \tilde{c}_{0}\left(\xi_{1}, t_{2}\right)-\frac{1}{2}\left(S_{1} \tilde{c}_{0}\right)\left(\xi_{1}, t_{2}\right)-\frac{1}{2}\left(S_{2} \tilde{c}_{0}\right)\left(\xi_{1}, t_{2}\right)+\left(S_{1} S_{2} \tilde{c}_{0}\right)\left(\xi_{1}, t_{2}\right) ; \\
& \tilde{C}_{2}\left(\xi_{1}, t_{3}\right)=\frac{1}{4} \tilde{c}_{0}\left(\xi_{1}, t_{3}\right)-\frac{1}{2}\left(S_{1} \tilde{c}_{0}\right)\left(\xi_{1}, t_{3}\right)+\frac{1}{2}\left(S_{2} \tilde{c}_{0}\right)\left(\xi_{1}, t_{3}\right)-\left(S_{1} S_{2} \tilde{c}_{0}\right)\left(\xi_{1}, t_{3}\right) ; \\
& \tilde{C}_{3}\left(\xi_{1}, t_{2}\right)=\frac{1}{4} \tilde{c}_{0}\left(\xi_{1}, t_{2}\right)+\frac{1}{2}\left(S_{1} \tilde{c}_{0}\right)\left(\xi_{1}, t_{2}\right)-\frac{1}{2}\left(S_{2} \tilde{c}_{0}\right)\left(\xi_{1}, t_{2}\right)-\left(S_{1} S_{2} \tilde{c}_{0}\right)\left(\xi_{1}, t_{2}\right) ; \\
& \tilde{C}_{4}\left(\xi_{1}, t_{3}\right)=\frac{1}{4} \tilde{c}_{0}\left(\xi_{1}, t_{3}\right)+\frac{1}{2}\left(S_{1} \tilde{c}_{0}\right)\left(\xi_{1}, t_{3}\right)+\frac{1}{2}\left(S_{2} \tilde{c}_{0}\right)\left(\xi_{1}, t_{3}\right)+\left(S_{1} S_{2} \tilde{c}_{0}\right)\left(\xi_{1}, t_{3}\right) .
\end{aligned}
$$

Here we have used the change of variables $\xi_{2}-b \xi_{3}=t_{2}, \xi_{2}+b \xi_{3}=t_{3}$ and respectively

$$
\xi_{2}=\frac{t_{3}+t_{2}}{2}, \quad \xi_{3}=\frac{t_{3}-t_{2}}{2 b} \rightarrow 0, b \rightarrow \infty .
$$


Thus, according to the formula (4) we have for new variables $\xi_{1}, t_{2}, t_{3}, b \rightarrow \infty$

$$
\tilde{A}_{\neq}\left(\xi_{1}, \frac{t_{3}+t_{2}}{2}, 0\right) \tilde{u}\left(\xi_{1}, \frac{t_{3}+t_{2}}{2}, 0\right)=\frac{\tilde{c}_{0}\left(\xi_{1}, t_{3}\right)+\tilde{c}_{0}\left(\xi_{1}, t_{2}\right)}{2}+\left(S_{2} \tilde{c}_{0}\right)\left(\xi_{1}, t_{3}\right)-\left(S_{2} \tilde{c}_{0}\right)\left(\xi_{1}, t_{2}\right) .
$$

Using the condition (6) we write

$$
\tilde{A}_{\neq}\left(\xi_{1}, \frac{t_{3}+t_{2}}{2}, 0\right) \tilde{g}\left(\xi_{1}, \frac{t_{3}+t_{2}}{2}\right)=\frac{\tilde{c}_{0}\left(\xi_{1}, t_{3}\right)+\tilde{c}_{0}\left(\xi_{1}, t_{2}\right)}{2}+\left(S_{2} \tilde{c}_{0}\right)\left(\xi_{1}, t_{3}\right)-\left(S_{2} \tilde{c}_{0}\right)\left(\xi_{1}, t_{2}\right)
$$

and finally according to the formula (7)

$$
\begin{aligned}
\tilde{A}_{\neq} & \left(\xi_{1}, \frac{t_{3}+t_{2}}{2}, 0\right) \tilde{g}\left(\xi_{1}, \frac{t_{3}+t_{2}}{2}\right)=\frac{\tilde{A}_{\neq}\left(\xi_{1}, t_{3}, 0\right) \tilde{g}\left(\xi_{1}, t_{3}\right)+\tilde{A}_{\neq}\left(\xi_{1}, t_{2}, 0\right) \tilde{g}\left(\xi_{1}, t_{2}\right)}{2} \\
& +\frac{i}{2 \pi}\left(v \cdot p \cdot \int_{-\infty}^{+\infty} \frac{\tilde{A}_{\neq}\left(\xi_{1}, \eta, 0\right) \tilde{g}\left(\xi_{1}, \eta\right) d \eta}{t_{3}-\eta}-v \cdot p \cdot \int_{-\infty}^{+\infty} \frac{\tilde{A}_{\neq}\left(\xi_{1}, \eta, 0\right) \tilde{g}\left(\xi_{1}, \eta\right) d \eta}{t_{2}-\eta}\right) .
\end{aligned}
$$

\section{MAIN RESULTS}

Collecting our above evaluations we can formulate the following main result.

Theorem 3. Let $æ-s=1+\delta,|\delta|<1 / 2, g \in H^{s+1 / 2}\left(\mathbb{R}^{2}\right)$. Then for solving the Boundary value problem (3), (5) the following assertions are valid:

1. if $a=b=0$ then the unique solution is given by the formula (4), where $c_{0}$ is defined by the formula (7);

2. if $a \rightarrow 0, b=$ const then the unique solution is defined by the formula (8);

3. if $a=$ const, $b \rightarrow 0$ then the unique solution is defined by the formula (9);

4. if $a \rightarrow \infty, b=$ const then limit of the solution exists if and only if the function $g$ satisfies the equation (10);

5. if $a=$ const, $b \rightarrow \infty$ then limit of the solution exists if and only if the function $g$ satisfies the equation (11).

APPENDIX

\section{A PRIORI ESTIMATES}

For first three cases 1, 2, 3 of Theorem 3 we can give an a priori estimate for the solution using the following lifting lemma; it was proved in [17].

Lemma 2. If $d\left(x_{1}, x_{2}\right) \in H^{s}\left(\mathbb{R}^{2}\right)$ then $D\left(x_{1}, x_{2}, x_{3}\right)=d\left(x_{1}+a x_{3}, x_{2}+b x_{3}\right)$ belongs to the space $H^{s-1 / 2}\left(\mathbb{R}^{3}\right), a, b \geq 0, a^{2}+b^{2} \neq 0$.

To distinct the norms in $\mathbb{R}^{3}$ and $\mathbb{R}^{2}$ we will the notations $\|\cdot\|_{s}$ and $[\cdot]_{s}$.

Theorem 4. Let $-1 / 2<\delta<0$. The the boundary value problem (3), (5) has a unique solution for an arbitrary $g \in H^{s+1 / 2}\left(\mathbb{R}^{2}\right)$ in the space $H^{s}\left(C_{+}^{a b}\right)$. This solution can be constructed explicitly by the Fourier transform and the one-dimensional singular integral operator. The a priori estimate $\|u\|_{s} \leq c[g]_{s+1 / 2}$ holds.

Proof. 1. The case 1. We have

$$
\tilde{u}(\xi)=\frac{\tilde{A} \neq\left(\xi^{\prime}, 0\right)}{\tilde{A}_{\neq}(\xi)} \tilde{g}\left(\xi_{1}, \xi_{2}\right)
$$


Then

$$
\begin{gathered}
\|u\|_{s}^{2}=\int_{\mathbb{R}^{3}}\left|\frac{\tilde{A} \neq\left(\xi^{\prime}, 0\right)}{\tilde{A}_{\neq}(\xi)} \tilde{g}\left(\xi_{1}, \xi_{2}\right)\right|^{2}(1+|\xi|)^{2 s} d \xi \leq \int_{\mathbb{R}^{3}} \frac{\left(1+\left|\xi^{\prime}\right|\right)^{2 æ}(1+|\xi|)^{2 s}}{(1+|\xi|)^{2 æ}}\left|\tilde{g}\left(\xi_{1}, \xi_{2}\right)\right|^{2} d \xi \\
\leq C \int_{\mathbb{R}^{2}}\left(1+\left|\xi^{\prime}\right|\right)^{2 s+1}\left|\tilde{g}\left(\xi_{1}, \xi_{2}\right)\right|^{2} d \xi^{\prime}=C[g]_{s+1 / 2 .}
\end{gathered}
$$

The cases 2, 3. Here, we need to use Lemma 2 and estimates for one-dimensional singular integral operators $S_{1}, S_{2}$. Since $h\left(\xi^{\prime}\right) \equiv \tilde{A}_{\neq}\left(\xi^{\prime}, 0\right) \tilde{g}\left(\xi^{\prime}\right) \in \widetilde{H}^{s+1 / 2-\circledast}\left(\mathbb{R}^{2}\right) \equiv H^{s_{0}}\left(\mathbb{R}^{2}\right)$ we have $s_{0}=$ $s-\mathfrak{x}+1 / 2=-1 / 2-\delta$, so that $-1 / 2<s_{0}<0$. Then we obtain $\left[S_{k} \tilde{h}\right]_{s_{0}} \leq c[\tilde{h}]_{s_{0}}, k=1,2$, because the operators $S_{1}, S_{2}$ are bounded in $\widetilde{H}^{s_{0}}\left(\mathbb{R}^{2}\right)$ [7]. Finally, $[\tilde{h}]_{s_{0}} \leq c[\tilde{g}]_{s+1 / 2}$ according to Lemma 2 and properties of pseudo-differential operators.

Remark 2. The author's paper [17] has some inaccuracies in subsections $7.4,7.5$, the author took into account the wrong sign for one of summands in the general solution. Really, the formula is more simple and the a priori estimates also. This paper gives right signs in the formula for a general solution.

\section{CONCLUSION}

The author hopes that these considerations will be continued for more complicated multidimensional situations [18-21], and it can be useful for the theory of boundary value problems in domains with nonsmooth boundaries.

\section{REFERENCES}

1. Ju. V. Egorov and B.-W. Schulze, Pseudo-Differential Operators, Singularities, Applications (Birkhäuser, Basel, 1997).

2. V. E. Nazaikinskii, A. Yu. Savin, B.-W. Schulze, and B. Yu. Sternin, Elliptic Theory on Singular Manifolds (Chapman and Hall/CRC, Boca Raton, 2006).

3. S. A. Nazarov and B. A. Plamenevsky, Elliptic Problems in Domains with Piecewise Smooth Boundaries (Walter de Gruyter, Berlin, New York, 1994).

4. B.-W. Schulze, Boundary Value Problems and Singular Pseudo-Differential Operators (Wiley, Chichester, 1998).

5. B.-W. Schulze, B. Sternin, and V. Shatalov, Differential Equations on Singular Manifolds; Semiclassical Theory and Operator Algebras (Wiley, Berlin, 1998).

6. G. I. Eskin, "A conjugacy problem for equations of principal type with two independent variables," Tr. Mosk. Mat. Ob-va 21, 245-292 (1970).

7. G. Eskin, Boundary Value Problems for Elliptic Pseudodifferential Equations (AMS, Providence, RI, $1981)$.

8. S. G. Milkhin and S. Prößdorf, Singular Integral Operators (Akademie, Berlin, 1986).

9. V. S. Vladimiriv, Methods of the Theory of Generalized Functions (Taylor and Francis, London, 2002).

10. F. D. Gakhov, Boundary Value Problems (Dover, Mineola, NY, 1981).

11. N. I. Muskhelishvili, Singular Integral Equations (North-Holland, Amsterdam, 1976).

12. V. B. Vasil'ev, Wave Factorization of Elliptic Symbols: Theory and Applications. Introduction to the Theory of Boundary Value Problems in Non-Smooth Domains (Kluwer Academic, Dordrecht, Boston, London, 2000).

13. V. B. Vasilyev, "On the Dirichlet and Neumann problems in multi-dimensional cone," Math. Bohem. 139, 333-340 (2014).

14. V. B. Vasilyev, "On certain elliptic problems for pseudo differential equations in a polyhedral cone," Adv. Dyn. Syst. Appl. 9, 227-237 (2014).

15. V. B. Vasil'ev, "Pseudodifferential equations on manifolds with complicated boundary singularities," J. Math. Sci. 230, 175-183 (2018). 
16. V. B. Vasilyev, "Pseudo-differential equations and conical potentials: 2-dimensional case," Opusc. Math. 39, 109-124 (2019).

17. V. B. Vasilyev, "Pseudo-differential equations, wave factorization, and related problems," Math. Meth. Appl. Sci. 41, 9252-9263 (2018).

18. V. B. Vasilyev, "Pseudo-differential operators on manifolds with a singular boundary," in Modern Problems in Applied Analysis, Ed. by P. Drygas and S. Rogosin (Birkhäuser, Cham, 2018), pp. 169-179.

19. V. B. Vasilyev, "Asymptotical analysis of singularities for pseudo differential equations in canonical nonsmooth domains," in Integral Methods in Science and Engineering. Computational and Analytic Aspects, Ed. by C. Constanda and P. J. Harris (Birkhäuser, Boston, 2011), pp. 379-390.

20. V. B. Vasil'ev, "Pseudodifferential equations in cones with conjugate points on the boundary," Differ. Equat. 51, 1113-1125(2015).

21. V. B. Vasilyev, "On the asymptotic expansion of certain plane singular integral operators," Boundary Value Probl. 116, 1-13 (2017). 